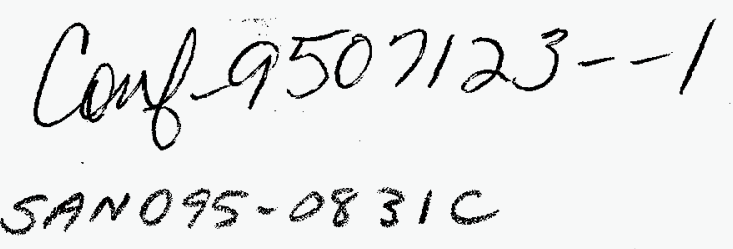

\title{
Minimizing Phylogenetic Number to find Good Evolutionary Trees
}

Leslie Ann Goldberg ${ }^{1}$, Paul W. Goldberg ${ }^{1}$, Cynthia A. Phillips ${ }^{1}$, Elizabeth Sweedyk $^{2}$ and Tandy Warnow ${ }^{3}$

1 Sandia National Laboratories, MS 1110, P.O. Box 5800, Albuquerque, NM 87185 , U.S.A.

2593 Soda Hall, Dept. of Computer Science, UC Berkeley, Berkeley, CA 94720, U.S.A.

3 Dept. of Computer and Information Science, University of Pennsylvania. Philadelphia, PA 19104, U.S.A.

\begin{abstract}
Inferring phylogenetic trees is a fundamental problem in computational-biology. We present a new objective criterion, the phylogenetic number, for evaluating evolutionary trees for species defined by biomolecular sequences or other qualitative characters. The phylogenetic number of a tree $T$ is the maximum number of times that any given character state arises in $T$. By contrast, the classical parsimony criterion measures the total number of times that different character states arise in $T$. We consider the following related problems: finding the tree with minimum phylogenetic number, and computing the phylogenetic number of a given topology in which only the leaves are labeled by species. When the number of states is bounded (as is the case for biomolecular sequence characters), we can solve the second problem in polynomiai time. We can also compute a fixed-topology 2-phylogeny (when one exists) for an arbitrary number of states. This algorithm can be used to further distinguish trees that are equal under parsimony. We also consider a number of other related problems.
\end{abstract}

\section{Introduction}

The problem of evolutionary tree construction involves taking a given set of species, and constructing a tree which describes the evolutionary history of that set of species. We would expect a pair of species to be close together in the tree if they are closely related. Numerous variants of this general problem have been studied, the variants arising from the differing kinds of information that may be assumed to be available concerning the species.

In character-based phylogeny, the scenario is the following. A character $c$ is a function from the species set $S$ to some set $R_{c}$ of states. For example, the character vertebrate-invertebrate has two states, so we can choose $R_{c}=\{0,1\}$ and we 
can define $c$ so that $c(s)=0$ for every species $s$ that is a vertebrate and $c(s)=$ 1 for every species $s$ that is an invertebrate. As another example, we could define a character $c$ based on average life-span. In this case $R_{c}$ might be a set of ranges such as $R_{c}=\{0-10$ years, $10-20$ years, 20-60 years, more than 60 years $\}$. Then the function $c$ could be defined to map each species $s$ to the range containing its average life-span. We can think of a sequence of $k$ characters $c_{1}, \ldots, c_{k}$ as mapping each species $s$ in the species set to a vector $\left(c_{1}(s), \ldots, c_{k}(s)\right)$ in $R_{c_{1}} \times \cdots \times R_{c_{k}}$. The species sets that we will consider will have the property that for any two distinct species, $s$ and $s^{\prime}$, that are in a species set, $\left(c_{1}(s), \ldots, c_{k}(s)\right) \neq\left(c_{1}\left(s^{\prime}\right), \ldots, c_{k}\left(s^{\prime}\right)\right)$. Thus, we will be able to identify each species $s$ with a vector $\left(c_{1}(s), \ldots, c_{k}(s)\right)$ in $R_{c_{1}} \times \cdots \times R_{c_{k}}$. Furthermore, we will think of the set $R_{c_{1}} \times \cdots \times R_{c_{k}}$ as being the set containing all possible species, including those in $S$.

The inputs to the phylogeny construction problem are the species set $S$ (we will use $n$ to denote the size of $S$ ) and a sequence of characters, $c_{1}, \ldots, c_{k}$. We will let $r_{c_{j}}$ denote $\left|R_{c_{j}}\right|$, and $r$ denote $\max _{j} r_{c_{j}}$. A phylogenetic tree for the input is a node-labeled tree in which every node of the tree is labeled with a vector in $R_{c_{1}} \times \cdots \times R_{c_{k}}$, and each species in $S$ is the label of some node of the treet. Thus, each character $c_{j}$ can be extended to a function from the set of vertices of $T$ to $R_{c_{j}}$.

We can think of a species as a string of length $k$ over the alphabet $\{1, \ldots, r\}$. A phylogeny is a way of expressing similarity amongst a set of strings rather than expressing similarity between pairs of strings. Strings which have strong similarities (as measured by matches in many locations) are located closer to each other in the tree than those that are more disparate. The output tree is the pattern of similarity amongst the entire set of input strings.

The measure of fitness of a phylogenetic tree is computed in one of several ways. Classically, there have been essentially two measures of fitness. The first is parsimony, in which a tree is sought such that the total weight of the tree is minimized, where the weight of the tree is the sum of the edge-weights, and the weight of an edge is the number of characters on which the species at its endpoints disagree. The other criterion is called compatibility, in which a tree is sought to maximize the number of characters that are convex on the tree. A character $c_{j}$ is convex on a tree $T$ if for each state $i \in R_{c_{j}}, c_{j}^{-1}(i)$ defines a connected component of $T$.

† A phylogenetic tree for the input $S, c_{1}, \ldots, c_{k}$ is sometimes defined to be a node-labeled tree in which every node of the tree is labeled with a vector in $R_{c_{1}} \times \cdots \times R_{c_{k}}$, and each species in $S$ is the label of some leaf of the tree. It is clear that every tree satisfying this alternative definition also satisfies our definition above. The alternative definition is equivalent to ours in the sense that we can convert a tree $T$ satisfying our definition into a tree $T^{\prime}$ satisfying the alternative definition by adding extra leaves. Under all reasonable measures of fitness for phylogenetic trees, $T$ and $T^{\prime}$ will have the same measure of fitness. 


\section{DISCLAIMER}

This report was prepared as an account of work sponsored by an agency of the United States Government. Neither the United States Government nor any agency thereof, nor any of their employees, make any warranty, express or implied, or assumes any legal liability or responsibility for the accuracy, completeness, or usefuiness of any information, apparatus, product, or process disclosed, or represents that its use would not infringe privately owned rights. Reference herein to any specific commercial product, process, or service by trade name, trademark, manufacturer, or otherwise does not necessarily constitute or imply its endorsement, recommendation, or favoring by the United States Government or any agency thereof. The views and opinions of authors expressed herein do not necessarily state or reflect those of the United States Government or any agency thereof. 


\section{DISCLAIMER}

Portions of this document may be illegible in electronic image products. Images are produced from the best available original document. 
Both criteria, compatibility and parsimony, result in NP-hard optimization problems $[4,5]$. An ideal tree is one in which al! characters are compatible (i.e., all characters are convex on the tree). Such a tree is optimal under parsimony and compatibility criteria and is called a perfect phylogeny. The question of whether a perfect phylogeny exists for a given input is NP-Complete $[3,14]$.

In this paper, we consider an alternative measure of fitness which combines some of the properties of compatibility and parsimony. We will say that a phylogenetic tree $T$ for an input consisting of a species set $S$ and a sequence of characters $c_{1}, \ldots, c_{k}$ is an $\ell$-phylogeny if, for every character $c_{j}$ and every state $i \in R_{c_{j}}$, the set of vertices $c_{j}^{-1}(i)$ form at most $\ell$ connected components in $T$. (A 1-phylogeny is the same as a perfect phylogeny). The $\ell$-phylogeny problem is the problem of determining whether an input has an $\ell$-phylogeny. The phylogenetic number of an input is the minimum $\ell$ such that the input has an $\ell$-phylogeny. The phylogenetic number problem is the problem of determining the phylogenetic number of an input.

The $\ell$-phylogeny problem and the phylogenetic number problem both have fixed-topology versions which are defined as follows. The input is a species set $S$, a sequence of characters $c_{1}, \ldots, c_{k}$, and a tree $T$ in which internal nodes are unlabeled and each leaf is labeled with a species $s \in S$. Each species $s \in S$ is the label of exactly one leaf of $T$. A phylogenetic tree for the input is formed by taking $T$ and labeling the internal nodes of $T$ with vectors in $R_{c_{1}} \times \cdots \times R_{c_{k}}$. The fixed-topology $\ell$-phylogeny problem is the problem of determining whether the input has an $\ell$-phylogeny. The fixed-topology phylogenetic number problem is defined analogously.

The $\ell$-phylogeny problem and the phylogenetic number problem also have restricted versions in which new ancestral species may not be added. The restricted versions are defined as follows. The input is a species set $S$ and a sequence of characters $c_{1}, \ldots, c_{k}$. A restricted phylogenetic tree for the input is a node-labeled tree in which every node of the tree is labeled with a vector in $S$, and each species in $S$ is the label of some node of the tree. The restricted $\ell$-phylogeny problem is the problem of determining whether the input has a restricted $\ell$-phylogeny. The restricted phylogenetic number problem is defined analogously.

The $\ell$-phylogeny problem can be generalized as follows. Fix positive integers $r, \ell_{1}, \ldots, \ell_{r}$. Suppose that $S, c_{1}, \ldots, c_{k}$ is a phylogeny input such that $\max _{j} r_{c_{j}} \leq r$. An $\left(\ell_{1}, \ldots, \ell_{r}\right)$-phylogeny for an input is defined to be a phylogenetic tree for the input such that, for each character $c_{j}$ and each integer $i \leq\left|R_{c_{j}}\right|$, the set of vertices that are mapped to the $i$ th state in $R_{c_{j}}$ by $c_{j}$ forms at most $\ell_{i}$ connected components in $T$. The $\left(\ell_{1}, \ldots, \ell_{r}\right)$-phylogeny problem is the problem of determining whether an input has an $\left(\ell_{1}, \ldots, \ell_{r}\right)$ phylogeny. A generalized version of the restricted $\ell$-phylogeny problem is defined analogously. 


\subsection{Summary of Results and Outline of Paper:}

The 1-phylogeny problem is also known as the perfect phylogeny problem. It was shown to be NP-hard by Bodlaender, Fellows, Warnow, and (independently) Steel $[3,14]$. The hardness of 1-phylogeny implies that the phylogenetic number problem is NP-hard. In Section 2 of this paper we show that for any fixed $\ell>1$ the $\ell$-phylogeny problem is also NP-hard.

Having shown that the $\ell$-phylogeny problem is NP-hard, we consider in Section 3 the fixed topology $\ell$-phylogeny problem. It is known that the fixedtopology 1-phylogeny problem can be solved in polynomial time [7]. We show that the fixed-topology 2-phylogeny problem can also be solved in polynomial time and that the fixed-topology $\ell$-phylogeny problem is NP-hard for fixed $\ell>2$. (We show that the fixed-topology $\ell$-phylogeny problem is NP-hard for fixed $\ell>2$ even when the input is guaranteed to have an $\ell+1$-phylogeny and the degree of the topology is restricted to be at most 3.)

In Section 4 we consider the restricted $\ell$-phylogeny problem. We show that there is a polynomial-time algorithm for the restricted 1-phylogeny problem, but the restricted $\ell$-phylogeny problem is NP-hard for fixed $\ell \geq 2$.

Although the 1-phylogeny problem is NP-hard, it can be solved in polynomial time if the number, $n$, of species is fixed, or the number, $k$, of characters is fixed $[12,1]$, or the quantity $r=\max _{j} r_{c_{j}}$ is fixed $[2,11]$. A full analysis of fixed parameter $\ell$-phylogeny problems is outside the scope of this paper. However, we observe that all of the phylogeny problems can be solved in polynomial time (by brute force) if $n$ is fixed. In Section 5 we use interesting combinatorial techniques to show that for $k=2$ the phylogenetic number problem can be solved in polynomial time. The complexity of the $\ell$-phylogeny problem remains open for fixed $\ell>1$ and fixed $k>2$. The difficulty of fixed-topology phylogeny problems cloes not change if $k$ is fixed. In Section 6 we show that the fixedtopology phylogenetic number problem can be solved in polynomial time for fixed $r$. On a related note, we show that if $r$ is fixed, there is a polynomial-delay algorithm for listing fixed-topology $\ell$-phylogenies. We also show that for fixed $r \geq 2$ and fixed $\ell \geq 3$ the restricted $\ell$-phylogeny problem is NP-hard. (This

result follows from a more general result. Namely, we show that the restricted $\left(\ell_{1}, \ell_{2}\right)$-phylogeny problem is NP-hard for fixed $\ell_{1} \geq 2$ and $\ell_{2} \geq 2$ as long as one of $\ell_{1}, \ell_{2}$ is greater than 2.)

Finally, in section 7 we offer some concluding remarks and present some open problems. 


\subsection{Preliminary Facts}

The following fact is used in some of the proofs and in the restricted 1-phylogeny algorithm.

Fact 1: If an input $S, c_{1}, \ldots, c_{k}$ has an $\ell$-phylogeny then it has an $\ell$-phylogeny in which:

1. Each leaf has a label from $S$.

2. Each species is the label of at most one node.

3. Every node whose label is not in $S$ has degree at least 3.

4. There are at most $\max (0, n-2)$ nodes with labels that are not in $S$.

Proof: It is easy to see that conditions 1-3 can be satisfied. (One can convert an $\ell$-phylogeny into one that satisfies conditions $1-3$ by removing leaves with labels that are not in $S$, combining branches of the tree to accomplish condition 2, and then "splicing out" the appropriate degree 2 nodes to accomplish condition 3.) To prove that condition 4 can also be satisfied, suppose that $T$ is an $\ell$-phylogeny for the input that satisfies conditions $1-3$ and contains at least one node, $w$, with a label that is not in $S$. Let $T^{\prime}$ be the tree obtained from $T$ by splicing out any nodes of degree 2. (Condition 3 guarantees that no node with a label outside of $S$ is spliced out in this process.) Consider $T^{\prime}$ to be rooted at $w$. It is easy to see that we can add one or more new internal nodes to $T^{\prime}$ to obtain a complete binary tree $T^{\prime \prime}$ which is rooted at $w$ and has the same leaves as $T^{\prime} \dagger$. Conditions 1 and 2 imply that $T$, and therefore $T^{\prime}$ and $T^{\prime \prime}$, have at most $n$ leaves. Since $T^{\prime \prime}$ has at most $n$ leaves, it has at most $n-1$ internal nodes. Therefore, $T^{\prime}$ has at most $n-2$ internal nodes, and $T$ has at most $n-2$ nodes with labels that are not in $S$. 口

Fact 1 implies that if an input has an $\ell$-phylogeny then it has a polynomialsized $\ell$-phylogeny.

$\dagger$ To see how to construct $T$ ", let the "level" of a vertex denote its distance from the root. Start with level 0 of $T^{\prime}$ and proceed through the levels of the tree in increasing order. Consider each vertex $v$ on each level. If $v$ has children $x_{1}, \ldots, x_{j}$ with $j>2$ remove the edges $\left(v, x_{2}\right), \ldots,\left(v, x_{j}\right)$ and add a new node $y$ which is a child of $v$ and the parent of nodes $x_{2}, \ldots, x_{j}$. Note that at least one new internal node is added in the process, as $w$ has at least three children in $T^{\prime}$. 


\section{The Hardness of $\ell$-Phylogeny}

In this section we show that for any fixed $\ell>1$, the $\ell$-phylogeny problem is NP-hard. Our reduction is from the 1-phylogeny problem, which was shown to be NP-hard by Bodlaender, Fellows, Warnow, and (independently) Steel $[3,14]$.

We define the weight of an edge $\left(v_{1}, v_{2}\right)$ in a phylogeny to be the number of characters $c_{j}$ such that $c_{j}\left(v_{1}\right) \neq c_{j}\left(v_{2}\right)$. That is, the weight of $\left(v_{1}, v_{2}\right)$ is the number of characters on which the species labeling $v_{1}$ and $v_{2}$ disagree. We define the weight of a phylogeny to be the sum of the weights of its edges. We start with the following observation.

Observation 2: Let $S, c_{1}, \ldots, c_{k}$ be any input to the $\ell$-phylogeny problem and let $r$ denote $\max _{j} r_{c_{j}}$. Any $\ell$-phylogeny for this input has weight at most $l(\ell r-1)$.

We will use the following lemma.

Lemma 3: For every integer $\ell$ there is an input $I_{\ell}=S, c_{1}, \ldots, c_{2 \ell}$ in which $|S|=2 \ell^{3}-2 \ell+1$ and $R_{c_{j}}=\{0, \ldots, \ell-1\}$ for $1 \leq j \leq 2 \ell$ such that

1. For every state $i$ in the range $0 \leq i<\ell$, the species $i^{2 \ell}$ is in $S$.

2. I $I_{\ell}$ has an $\ell$-phylogeny

3. In any $\ell$-phylogeny for $I_{\ell}$ the subgraph induced by all of the nodes with any given label is connected.

4. In any $\ell$-phylogeny for $I_{\ell}$ all of the nodes are labeled by species in $S$. (That is, no new species are introduced.)

5. In any $\ell$-phylogeny for $I_{\ell}$ the path between the species $i^{2 \ell}$ and $j^{2 \ell}$ for $i \neq j$ passes through at least $2 \ell-1$ distinct species.

Example: The Input $I_{3}$

The species set $S$ of input $I_{3}$ consists of 49 species. The values of the six characters on these species are defined as in figure 1: 
000000100000110000111000211000221000222000

000100000110000111000211000221000222

010000

010010

110010

111010

111110

111111211111212111212121012121010121010101

121111121211121212101212101012101010

112111

122111

222111

222211

222221

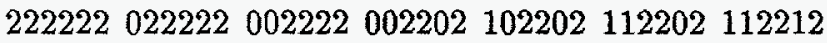

220222220022220020221020221120221121

Fig. 1. The Input $I_{3}$

The Input $I_{3}$ has a 3 -phylogeny in which species are connected along the rows and along the first column. Each node $i^{6}$ is also connected to the row beneath it. By Observation 2 any 3-phylogeny for $I_{3}$ has weight at most 48 . However, 48 edges with positive weight are needed just to hook up the 49 species in $S$ into a tree. We conclude that any 3-phylogeny for $I_{3}$ consists of 48 edges with weight 1 plus possibly some edges with weight 0 . Thus, the subgraph induced by all of the nodes with any given label forms a single connected component. Furthermore, no new species are introduced. Finally, since $i^{6}$ and $j^{6}$ differ in 6 characters, any path between them in any 3-phylogeny for $I_{3}$ passes through at least 5 distinct species.

Construction of $I_{\ell}=S, c_{1}, \ldots, c_{2 \ell}$ :

For $1 \leq j \leq 2 \ell$ we set $R_{c_{j}}=\{0, \ldots, \ell-1\}$. For each state $i$ in the range $0 \leq i<\ell$ we put the species $i^{2 \ell}$ into $S$. The other species in $S$ will be the species in the following phylogeny:

For each state $i$ in the range $0 \leq i<\ell$ we will choose a unique partition $P_{i}$ of the $2 \ell$ characters into two sets of size $\ell$. (In the construction of $I_{3}$ above we used $P_{0}=\{0,1,2\},\{3,4,5\}, P_{1}=\{0,2,4\},\{1,3,5\}$, and $P_{2}=$ $\{0,1,4\},\{2,3,5\}$.)

We will use each of the parts of the partition $P_{i}$ to form a "row" of species which will be connected to the species $i^{2 \ell}$. To construct each row, consider the ordered list $c_{i_{1}}, \ldots, c_{i_{2}}$ consisting of the characters in the appropriate part of the partition. From the species $i^{2 \ell}$ form a new species by changing the state of character $c_{i_{1}}$ to $(i+1) \bmod \ell$. Then form a new species by changing the state of character $c_{i_{2}}$ to $(i+1) \bmod \ell$. Continue on until the state of character $c_{i_{\ell}}$ is 
changed to $(i+1) \bmod \ell$. Then change the state of character $c_{i_{1}}$ to $(i+2) \bmod$ $\ell$ and continue on in this manner until finally the state of character $c_{i \ell}$ is changed to $(i+(\ell-1)) \bmod \ell$.

Finally, we will add species to connect the species $i^{2 \ell}$ to the species $(i+1)^{2 \ell}$ in the vertical spine (for $i$ in the range $0 \leq i<\ell-1$ ). Let $c_{\lambda}$ be the second character in the first part of the partition corresponding to $i$ and construct a new species from $i^{2 \ell}$ by changing the state of character $c_{\lambda}$ to $i+1$. Next, let $c_{\lambda}^{\prime}$ be the first character such that $c_{\lambda}$ and $c_{\lambda}^{\prime}$ are in different parts of $i$ 's partition and $c_{\lambda}$ and $c_{\lambda}^{\prime}$ are in different parts of $(i+1)$ 's partition. Construct a new species by changing the state of character $c_{\lambda}^{\prime}$ to $i+1$. Now, construct $2 \ell-3$ more species by considering each remaining character in turn and changing it from state $i$ to state $i+1$.

Proof of Lemma 3: By construction, $S$ contains the species $i^{2 \ell}$ for every state $i$ in the range $0 \leq i<\ell$. To see that the phylogeny constructed above is indeed an $\ell$-phylogeny for $I_{\ell}$ note that for each state $i$ and for each state $j \neq i$ a character $c_{\lambda}$ only has state $i$ in one of the two rows connected to $j^{2 \ell}$ and the species with $c_{\lambda}$ in state $i$ are connected in this row. Furthermore, there is a single connected component with character $c_{\lambda}$ in state $i$ in the rows connected to $i^{2 \ell}$ and this connected component contains all species on the vertical spine with character $c_{\lambda}$ in state $i$. We now wish to show that all of the species introduced in the construction are distinct. Suppose that instead two species $s_{1}$ and $s_{2}$ have identical labels. Note that, by construction, $s_{1}$ and $s_{2}$ could not be of the form $i^{2 \ell}$. Furthermore, they could not be on the same horizontal row and they could not both be on the vertical spine. There are three cases to consider:

1. $s_{1}$ and $s_{2}$ are on different rows, both of which are attached to $i^{2 \ell}$.

In this case $s_{1}$ has state $i$ for all of the characters in one part of the partition $P_{i}$ and $s_{2}$ has state $i$ for all of the characters in the other part of the partition $P_{i}$ so it must be the case that $s_{1}=s_{2}=i^{2 \ell}$ which is a contradiction.

2. $s_{1}$ is on a horizontal row connected to $i^{2 \ell}$ and $s_{2}$ is on a horizontal row connected to $j^{2 \ell}$ for some $j \neq i$.

In this case $s_{1}$ has state $i$ for all of the characters in some part of the partition $P_{i}$ so $s_{2}$ must have character $i$ for all of the characters in that part of the partition $P_{i}$ and character $j$ on all other characters. But then the partition $P_{j}$ is the same as the partition $P_{i}$, which is not true by construction.

3. $s_{1}$ is on the vertical spine between $i^{2 \ell}$ and $(i+1)^{2 \ell}$ and $s_{2}$ is on a horizontal row.

By construction $s_{2}$ must be on a row attached to $i$ or on a row attached to $i+1$. However, the choice of $c_{\lambda}$ and $c_{\lambda}^{\prime}$ ensures that $s_{2}$ cannot be on either of these rows.

Now that we know that the species are distinct, we count them. There are $\ell$ species of the form $i^{2 \ell}$. Each of the $2 \ell$ horizontal rows has $\ell(\ell-1)$ species. Finally, there are $(\ell-1)(2 \ell-1)$ additional species on the vertical spine. We 
conclude that $S$ has $2 \ell^{3}-2 \ell+1$ distinct species. By Observation 2, any $\ell-$ phylogeny for $I_{\ell}$ has weight at most $2 \ell\left(\ell^{2}-1\right)=2 \ell^{3}-2 \ell$. However, $2 \ell^{3}-2 \ell$ edges with positive weight are needed just to hook up the $2 \ell^{3}-2 \ell+1$ species in $S$ into a tree. We conclude that any $\ell$-phylogeny for $I_{\ell}$ consists of $2 \ell^{3}-2 \ell$ edges with weight 1 plus possibly some edges with weight 0 . Thus, the subgraph induced by all of the nodes with any given label forms a single connected component. Furthermore, no new species are introduced. Finally, since $i^{2 \ell}$ and $j^{2 \ell}$ differ in $2 \ell$ characters, any path between them in any $\ell$-phylogeny for $I_{\ell}$ passes through at least $2 \ell-1$ distinct species. $\square$

We will use Lemma 3 to prove the following theorem

Theorem 4: For any fixed $\ell>1$ the $\ell$-phylogeny problem is NP-hard.

Proof: The reduction is from the 1-phylogeny problem. Let $S, c_{1}, \ldots, c_{k}$ be an input to the 1-phylogeny problem such that $R_{c_{j}}=\{0, \ldots, r-1\}$ for $1 \leq j \leq k$. Let $S^{\prime}, c_{1}^{\prime}, \ldots, c_{2 \ell}^{\prime}$ be an input to the $\ell$-phylogeny problem satisfying the conditions in Lemma 3. Let $S^{*}=\left\{s r^{k} \mid s \in S^{\prime}\right\}$. For each $i$ in the range $0 \leq i<\ell$ let $S_{i}=\left\{i^{2 \ell} y \mid y \in S\right\}$. Let $S^{\prime \prime}=S^{*} \cup \bigcup_{0 \leq i<\ell} S_{i}$. Let $I$ be the input to the $\ell$-phylogeny problem with species set $S^{\prime \prime}$ and characters $c_{1}^{\prime}, \ldots, c_{2 \ell}^{\prime}, c_{1}, \ldots, c_{k}$. (Note that in input $I$ the range of $c_{j}$ has been extended from $R_{c_{j}}$ to $R_{c_{j}} \cup\{r\}$.)

$\rightarrow$ Suppose that $T$ is a 1-phylogeny for $S, c_{1}, \ldots, c_{k}$. For each $i$ in the range $0 \leq i<\ell$ let $T_{i}$ be a copy of $T$ in which each label $y$ has been changed to $i^{2 \ell} y .\left(T_{i}\right.$ is a 1 -phylogeny for $S_{i}, c_{1}^{\prime}, \ldots, c_{2 \ell}^{\prime}, c_{1}, \ldots, c_{k}$.) Let $T^{*}$ be an $\ell$ phylogeny for $S^{*}, c_{1}^{\prime}, \ldots, c_{2 \ell}^{\prime}, c_{1}, \ldots, c_{k}$. (Part 2 of Lemma 3 guarantees that $T^{*}$ exists.) Now for each $i$ in the range $0 \leq i<\ell$ connect an arbitrary node in $T_{i}$ to the node $i^{2 \ell} r^{k}$ in $T^{*}$. (The construction, together with Part 1 of Lemma 3 guarantees that there is a vertex of $T^{*}$ labeled $i^{2 \ell} r^{k}$.) It is easy to see that the resulting tree is an $\ell$-phylogeny for $I$.

$\leftarrow$ Suppose that $T$ is an $\ell$-phylogeny for $I$. If we restrict our attention to characters $c_{1}^{\prime}, \ldots, c_{2 \ell}^{\prime}$, we still have an $\ell$-phylogeny. Therefore, by Part 3 of Lemma 3 , the subgraph induced by all of the species which have some particular set of states for characters $c_{1}^{\prime}, \ldots, c_{2 \ell}^{\prime}$ is connected. We will use the notation $T_{i}$ to refer to the induced subtree of $T$ containing those species that have state $i$ for characters $c_{1}^{\prime}, \ldots, c_{2 \ell}^{\prime}$.

We claim that for any $j$ in the range $1 \leq j \leq k$ any path in $T$ between a node $t_{i} \in T_{i}$ and a node $t_{h} \in T_{h}$ (for $h \neq i$ ) contains some species $s$ with $c_{j}(s)=r$. Clearly, this claim implies that $T_{0}$ is a 1-phylogeny for $S_{0}, c_{1}^{\prime}, \ldots, c_{2 \ell}^{\prime}$, $c_{1}, \ldots, c_{k}$. Hence, $S, c_{1}, \ldots, c_{k}$ has a 1-phylogeny.

To prove the claim note that by Part 5 of Lemma 3 the path between $T_{i}$ and $T_{h}$ passes through $2 \ell-1$ nodes $v_{1}, \ldots, v_{2 \ell-1}$, no two of which agree on all of characters $c_{1}^{\prime}, \ldots, c_{2 \ell}^{\prime}$. By construction and by Part 1 of Lemma $3, S^{\prime \prime}$ contains the species $i^{2 \ell} r^{k}$ and by Part 3 of Lemma 3 it is part of $T_{i}$. Similarly, $S^{\prime \prime}$ contains the species $h^{2 \ell} r^{k}$ and it is part of $T_{h}$. Furthermore, (by construction and by Part 4 of Lemma 3), for each node $v_{m}, S^{\prime \prime}$ contains a species $v_{m}^{\prime}$ that agrees with $v_{m}$ on characters $c_{1}^{\prime}, \ldots, c_{2 \ell}^{\prime}$ and has characters $c_{1}, \ldots, c_{k}$ in 
state $r$. By Part 3 of Lemma $3 v_{m}^{\prime}$ is in the connected subgraph of $T$ induced by species which agree with $v_{m}$ on characters $c_{1}^{\prime}, \ldots, c_{2 \ell}^{\prime}$. Now suppose that none of $v_{1}, \ldots, v_{2 \ell-1}$ has character $c_{j}$ in state $r$. Then the sub-graph of $T$ induced by those nodes that have character $c_{j}$ in state $r$ has $2 \ell+1$ connected components, which contradicts the fact that $T$ is an $\ell$-phylogeny. $\square$

\section{The Fixed-Topology $\ell$-Phylogeny Problem}

It is known that the fixed-topology 1-phylogeny problem can be solved in polynomial time [7]. In Subsection 3.1, we show that the fixed-topology 2-phylogeny problem can also be solved in polynomial time. In Subsection 3.2 we show that the fixed-topology $\ell$-phylogeny problem is NP-hard for fixed $\ell>2$. (We show that the fixed-topology $\ell$-phylogeny problem is NP-hard for fixed $\ell>2$ even when the input is guaranteed to have an $\ell+1$-phylogeny and the degree of the topology is restricted to be at most 3.)

\subsection{The Fixed-Topology 2-Phylogeny Problem}

In this subsection, we show that the fixed-topology 2-phylogeny problem can be solved in polynomial time. The algorithm runs in time $O(n r k)$ where $n$ is the number of species, $r$ is the maximum number of states in any character, and $k$ is the number of characters. If a 2 -phylogeny exists, then our algorithm computes a labeling that achieves a 2-phylogeny.

Since the topology is fixed, the characters are independent and can be handled one at a time. We will now show how to compute the labels for a single character in time $O(n r)$, where in this case $r$ is the number of states for this character. The overall bound then follows.

Although the input tree is unrooted, for this algorithm, we root this tree from an arbitrary internal node. The choice of root does not affect the existence of a 2-phylogeny, but it may affect the labeling.

Let $T$ be the input tree with leaves labeled by states $1,2, \ldots, r$. Consider a single state $i$ and let $T_{i}$ be the subtree of tree $T$ consisting of all the leaves labeled $i$ and the unique set of paths connecting this set of leaves. For state $i$ to have a single connected component in tree $T$, every node in $T_{i}$ must be labeled $i$. For state $i$ to have at most two connected components, every node in tree $T_{i}$ with degree greater than 2 must be labeled $i$ (otherwise state $i$ would be split into at least 3 components). We call such nodes branch points of tree $T_{i}$. The branch points and the leaves already labeled $i$ are the forced points of tree $T_{i}$. At most one path of degree- 2 nodes between two forced points can be labeled something other than $i$.

We begin by computing $T_{i}$ for $i=1, \ldots, r$. Each branch point of $T_{i}$ is labeled as such, each path between two forced points is given a unique label, 
and each degree- 2 node in $T_{i}$ is labeled with its path label. Note that the root of tree $T_{i}$ need not be a branch point. If each node of tree $T$ is given a length- $r$ vector, then information for all $r$ trees $T_{i}$ can be stored on top of each other. For example, node $v$ could be a branch point for tree $T_{i}$ ( $i$ th slot of the vector indicates branch point), on the $l$ th path for tree $T_{j}$ (the $j$ th slot of the vector has the number $l$ ), and not in tree $T_{h}$ (the $h$ th slot is null). We can compute all $r$ trees in time $O(n r)$ using depth-first search.

The first phase of the algorithm (the forced phase) computes all forced labels. For each tree $T_{i}$, each branch point of $T_{i}$ is labeled $i$ and a pointer to the node is placed into a queue. If at any time we try to label a node that is already labeled with something else, then we stop and report that there is no 2-phylogeny for this topology.

Now all path conflicts have to be settled for the labeled nodes. We remove the first node from the queue. Suppose it is node $v$ and it is labeled $i$. If this node is also in path $l$ of tree $T_{j}$ for some $j \neq i$, then tree $T_{j}$ must give up path $l$. Once path $l$ is broken, then in order to achieve 2 connected components for state $j$, every other path in tree $T_{j}$ must be labeled $j$. We traverse tree $T_{j}$, clearing path $l$ (setting slot $j$ to null for all nodes on path $l$ of tree $T_{j}$ ) and labeling all other nodes $j$. If we attempt to label a node that is aiready labeled, then we stop. There can be no 2-phylogeny. Otherwise, the newly-labeled nodes are added to the queue. We do this for all paths that go through node $v$, then clear path conflicts on all the other nodes in the queue. Because each node can be labeled, enqueued, dequeued, and processed at most once, and each tree can be traversed at most once, this phase can be completed in time $O(n r)$.

The final phase completes the labeling of the tree. If we succeed in emptying the queue without encountering a fatal conflict, it is still possible that some nodes remain unlabeled. We show that there is always a 2-phylogeny. Let trees $T_{i}$ and $T_{j}$ be left undetermined by the forced phase of the algorithm. If the intersection of these two trees is empty, there is no conflict between them. Otherwise, the intersection is connected ${ }^{*}$ and contains exactly one path from each tree ${ }^{t}$. Furthermore, the root of one of the trees (possibly both) is in the intersection ${ }^{t}$. Suppose that the root of $T_{i}$ is contained in $T_{i} \cap T_{j}$. Then tree $T_{i}$ gives up the path through its root (if both roots are contained in $T_{i} \cap T_{j}$, one of the trees chosen arbitrarily will give up the path through its root). By the structure of the intersection, this clears the conflict between tree $T_{i}$ and $T_{j}$. We can solve all conflicts between pairs of trees in a similar manner. Since each tree

* If two nodes $v_{1}$ and $v_{2}$ are both in $T_{i}$ and both in $T_{j}$, then every node on the unique path in $T$ between $v_{1}$ and $v_{2}$ must also be in both trees.

$\dagger$ If the intersection contained pieces of two paths from tree $T_{i}$, then it must contain a branch point for tree $T_{i}$ and therefore tree $T_{j}$ would have been forced to relinquish a path and left completely determined by the forced phase.

$\ddagger$ Consider a node in the intersection. If its parent in $T$ is in the intersection, move up to it. Continue until some parent is no longer in the intersection. That node is the root of at least one of $T_{i}$ and $T_{j}$ 
was not forced to give up a path in the forced phase of the algorithm (otherwise it would have been fully determined then), it is free to give up one path in this phase. Each tree will give up at most one path, namely the one through its root. Therefore, all conflicts are resolved and we have a 2-phylogeny. This phase of the algorithm can be implemented in $O(n r)$ time by processing each remaining tree in order (determining whether it must relinquish the path through its root, and claiming all other paths).

Thus we have shown how to compute the labelings of the internal nodes of the input tree $T$ in time $O(n r)$ per character for an overall time of $O(n r k)$. Thus, we have proved the following theorem.

Theorem 5: The fixed-topology 2-phylogeny problem can be solved in polynomial time.

\subsection{The Fixed-Topology $\ell$-Phylogeny Problem for $\ell>2$}

In this subsection we prove the following theorem.

Theorem 6: The fixed-topology $\ell-p h y l o g e n y$ probiem is NP-hard for fixed $\ell>2$.

Proof: The proof is by reduction from 3SAT. Let $\ell>2$ be fixed. Suppose that we are given an input to 3SAT. We will show how to construct a one-character input $S, c, T$ to the fixed-topology $\ell$-phylogeny problem such that the phylogeny input has an $\ell$-phylogeny if and only if the input to $3 S A T$ is satisfiable.

The species set $S$, the set $R_{c}$ of states, and the character $c$ are constructed as follows. For each of the $n$ variables, $x$, in the satisfiability input we have states $\boldsymbol{s}_{\boldsymbol{x}}$ and $s_{\bar{x}}$ anc: species $s_{(x, 1)}, \ldots, s_{(x, \ell+1)}$ and $s_{(\bar{x}, 1)}, \ldots, s_{(\bar{x}, \ell+1)}$ where $c\left(s_{(x, j)}\right)=$ $s_{x}$ and $c\left(s_{(\bar{x}, j)}\right)=s_{\bar{x}}$. For each of the $m$ clauses, $C$, in the satisfiability input we have state $s_{C}$ and species $s_{(C, 1)}, \ldots, s_{(C, \ell+1)}$ where $c\left(s_{(C, j)}\right)=s_{C}$. For the $i$ th occurrence of the literal $x$ in the satisfiability input, we have state $s_{x_{i}}$ and species $s_{\left(x_{i}, 1\right)}, \ldots, s_{\left(x_{i}, \ell+1\right)}$ where $c\left(s_{\left(x_{i}, j\right)}\right)=s_{x_{i}}$. Similarly, for the $i$ th occurance of the literal $\bar{x}$ in the satisfiability input, we have state $s_{\bar{x}_{i}}$ and species $s_{\left(\bar{x}_{i}, 1\right)}, \ldots, s_{\left(\bar{x}_{i}, \ell+1\right)}$ where $c\left(s_{\left(\bar{x}_{i}, j\right)}\right)=s_{\bar{x}_{i}}$. Let $N$ denote $n(2 \ell-3)+$ $m(4 \ell-11)$. For each $h$ in the range $1 \leq h<N$ we have a state $s_{h}^{\prime}$ and species $s_{(h, 1)}^{\prime}, \ldots, s_{(h, \ell+1)}^{\prime}$ where $c\left(s_{(h, j)}^{\prime}\right)=s_{h}^{\prime}$.

We will show how to construct a tree $T$ in which internal nodes are unlabeled and each leaf is labeled with a species in $S$. Each species in $S$ will be the label of exactly one leaf of $T$. To construct $T$ we will first construct trees $T_{1}, \ldots, T_{N}$. Finally, we will hook $T_{i}$ to $T_{i+1}$ for $1 \leq i<N$

We start by showing how to hook tree $T_{i}$ to tree $T_{i+1}$. Let $t_{i}$ be an internal node in $T_{i}$ of degree at most 2 and let $t_{i+1}$ be an internal node in $T_{i+1}$ of degree at most 2 (it will be clear from the construction that such small degree internal nodes exist in $T_{i}$ and $\left.T_{i+1}\right)$. Connect $t_{i}$ and $t_{i+1}$ with a chain of $\ell+1$ new internal nodes. Finally, give each of the internal nodes in the chain a leaf and 
label the new leaves with the species $s_{(i, 1)}^{\prime}, \ldots, s_{(i, \ell+1)}^{\prime}$. For example, if $\ell=3$ then connect $t_{i}$ and $t_{i+1}$ as in figure 2 :

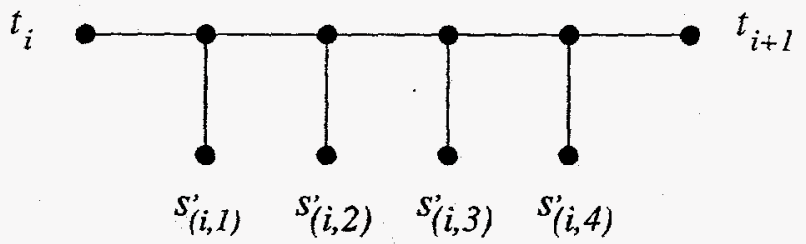

Fig. 2. Example for $\ell=3$

Note that in any $\ell$-phylogeny for the input, at least one of the internal nodes in the chain will be labeled with a species $s$ such that $c(s)=s_{i}^{\prime}$. Since we have now used all $\ell+1$ species $s$ with $c(s)=s_{i}^{\prime}$, neither $T_{i}$ nor $T_{i+1}$ contains a leaf $s$ such that $c(s)=s_{i}^{\prime}$. Therefore when $T_{i}$ is hooked to $T_{i+1}$ as above, any leaves $\ell_{i} \in T_{i}$ and $\ell_{i+1} \in T_{i+1}$ with $c\left(\ell_{i}\right)=c\left(\ell_{i+1}\right)$ are in different connected components in the subgraph induced by $c^{-1}\left(c\left(\ell_{i}\right)\right)$.

We next show how to construct the trees $T_{1}, \ldots, T_{N}$. Trees $T_{1}, \ldots, T_{N-n-m}$ will each consist of a single internal node connected to a single leaf. In particular, we will construct one such tree for each of the following species: for each variable $x$, species $s_{(x, 1)}, \ldots, s_{(x, \ell-2)}$ and $s_{(\bar{x}, 1)}, \ldots, s_{(\bar{x}, \ell-2)}$; for each clause $C$, species $s_{(C, 1)}, \ldots, s_{(C, \ell-3)}$; for the $i$ th occurance of the literal $x$, species $s_{\left(x_{i}, 1\right)}, \ldots, s_{\left(x_{i}, \ell-3\right)}$; for the $i$ th occurance of the literal $\bar{x}$, species $s_{\left(\bar{x}_{i}, 1\right)}, \ldots, s_{\left(\bar{x}_{i}, \ell-3\right)}$.

Trees $T_{N-n-m+1}, \ldots, T_{N-m}$ will be used for truth-setting. For each variable $x$ in the satisfiability input we will construct a tree as follows. Suppose that the literal $x$ appears $i$ times in the satisfiability input and that the literal $\bar{x}$ appears $j$ times in the satisfiability input. Construct a tree consisting of a chain of $2 i+2 j+6$ internal nodes. Each internal node will have one leaf, and the species at the leaves will be (in order): first, $s_{(x, \ell-1)}$; then, $s_{\left(x_{1}, \ell-2\right)}, s_{\left(x_{1}, \ell-1\right)}, s_{\left(x_{2}, \ell-2\right)}, s_{\left(x_{2}, \ell-1\right)}, \ldots, s_{\left(x_{i}, \ell-2\right)}, s_{\left(x_{i}, \ell-1\right)} ;$ then $s_{(x, \ell)}, s_{(\bar{x}, \ell-1)}$, $s_{(x, \ell+1)}, s_{(\bar{x}, \ell)}$; then $s_{\left(\bar{x}_{1}, \ell-2\right)}, s_{\left(\bar{x}_{1}, \ell-1\right)}, \ldots, s_{\left(\bar{x}_{j}, \ell-2\right)}, s_{\left(\bar{x}_{j}, \ell-1\right)}$; finally, $s_{(\bar{x}, \ell+1)}$. For example, if $\ell=3, i=1$, and $j=2$ construct a tree as in figure 3 :

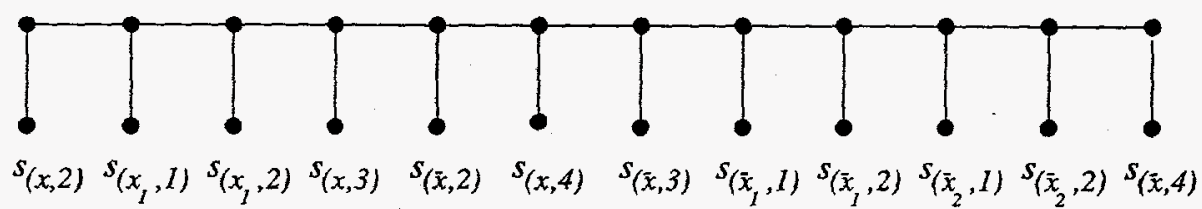

Fig. 3. Example for $\ell=3, i=1, j=2$ 
Because we have already introduced single-leaf trees for the species $s_{(x, 1)}, \ldots$, $s_{(x, \ell-2)}$ and $s_{(\bar{x}, 1)}, \ldots, s_{(\bar{x}, \ell-2)}$, we observe that in any $\ell$-phylogeny, the truthsetting tree for variable $x$ must have at most 2 connected components for each of the states $s_{x}$ and $s_{\bar{x}}$. We will say that an $\ell$-phylogeny sets the satisfiability variable $x$ to "true" if and only if the leaves $s_{(x, \ell)}$ and $s_{(x, \ell+1)}$ are in the same connected component for state $s_{x}$. If the variable $x$ is set to "true" then the leaf $s_{(x, \ell-1)}$ can be in a different connected component for state $s_{x}$. Therefore, for $1 \leq h \leq i$, state $s_{x_{h}}$ can form a single connected component in the truthsetting tree for $x$. Otherwise, state $s_{x_{h}}$ must have two connected components in the truth-setting tree for $x$. Similarly, if $x$ is set to "false" then leaves $s_{(\bar{x}, \ell-1)}$ and $s_{(\bar{x}, \ell)}$ can be in the same connected component for state $s_{\bar{x}}$ and leaf $s_{(\bar{x}, \ell+1)}$ can be in a different connected component. Therefore, for $1 \leq h \leq j$, state $s_{\bar{x}_{h}}$ can form a single connected component in the truth-setting tree for $x$. Otherwise, state $s_{\bar{x}_{k}}$ must have two connected components in the truth-setting tree for $x$.

Trees $T_{N-m+1}, \ldots, T_{N}$ will be used for clause-checking. For each clause $C=$ $x_{i} \vee \bar{y}_{j} \vee z_{k}$ in the satisfiability input we will construct a tree consisting of a chain of 10 internal nodes. Each internal node will have one leaf, and the species at the leaves will be (in order): $s_{(C, \ell-2)}, s_{\left(x_{i}, \ell\right)}, s_{\left(x_{i}, \ell+1\right)}, s_{(C, \ell-1)}, s_{\left(\bar{y}_{j}, \ell\right)}, s_{\left(\bar{y}_{j}, \ell+1\right)}, s_{(C, \ell)}$, $s_{\left(z_{k}, \ell\right)}, s_{\left(z_{k}, \ell+1\right)}, s_{(C, \ell-1)}$. For example, if $\ell=3$, construct a tree as in figure 4:

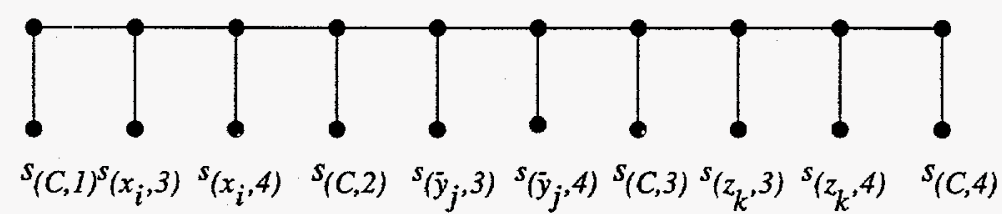

Fig. 4. Example for $\ell=3$

Because we have already introduced single-leaf trees for the species $s_{(C, 1)}, \ldots$, $s_{(C, \ell-3)}$, we observe that in any $\ell$-phylogeny, the clause-checking component for clause $C$ must have at most 3 connected components for the state $s_{C}$. This is possible if one of the literals in the clause has been set to "true" by the truth checking component and not otherwise. The correctness of the reduction follows. ㅁ

The input to the fixed-topology $\ell$-phylogeny problem that is constructed in the proof of Theorem 6 had two notable features. First, (because there are only $\ell+1$ species with each state), the input is guaranteed to have an $\ell+1$ phylogeny. Second, the degree of the tree $T$ is at most 3 . Therefore, the fixedtopology $\ell$-phylogeny problem is NP-hard for fixed $\ell>2$ even when the input is guaranteed to have an $\ell+1$-phylogeny and the degree of the topology is restricted to be at most 3 . 


\section{The Restricted $\ell$-Phylogeny Problem}

In this section we show that there is a polynomial time algorithm for the restricted 1-phylogeny problem. We then show that the restricted $\ell$-phylogeny problem is NP-hard for fixed $\ell \geq 2$.

We start by describing the algorithm for solving the restricted 1-phylogeny problem. Suppose that $S, c_{1}, \ldots, c_{k}$ is an input to the restricted 1-phylogeny problem. If the input has a restricted 1-phylogeny, it has one in which each species in $S$ is the label of exactly one node (if not, combine branches).

We define the weight of an edge $\left(v_{1}, v_{2}\right)$ in a phylogeny to be the number of characters $c_{j}$ such that $c_{j}\left(v_{1}\right) \neq c_{j}\left(v_{2}\right)$. That is, the weight of $\left(v_{1}, v_{2}\right)$ is the number of characters on which the species labeling $v_{1}$ and $v_{2}$ disagree. We define the weight of a phylogeny to be the sum of the weights of its edges.

Let $G$ denote the complete graph with vertex set $S$. We seek a spanning tree $T$ of $G$ in which, for every character $c_{j}$ and every state $i \in R_{c_{j}}$, the set of vertices $c_{j}^{-1}(i)$ form a connected component in $T$. Let the weight of an edge $\left(s, s^{\prime}\right)$ in $G$ be the number of characters $c_{j}$ such that $c_{j}(s) \neq c_{j}\left(s^{\prime}\right)$. It is easy to see that a spanning tree of $G$ is a 1-phyiogeny for the input if and only if its weight is $\sum_{j=1}^{k}\left(r_{c_{j}}-1\right)$. Therefore, the restricted 1-phylogeny problem reduces to the minimum weight spanning tree problem, which can be solved in polynomial time [13]. We have proved the following theorem

Theorem 7: The restricted 1-phylogeny problem can be solved in polynomial time.

In the remainder of this section, we prove the following theorem.

Theorem 8: The restricted $\ell$-phylogeny problem is $N P$-hard for fixed $\ell \geq 2$.

Proof: The reduction is from the $\ell$-consecutive ones problem, which is defined as follows:

INSTANCE: A $(0,1)$-matrix $M$.

QUESTION: Can the rows of $M$ be permuted in such a way that for each column in the resulting matrix, there are at most $\ell$ sequences of consecutive ones.

The $\ell$-consecutive ones problem is known to be solvable in polynomial time for $\ell=1$ [8]. However, it is NP-complete for fixed $\ell>1$ [9].

Let $\ell$ be a positive integer that is greater than or equal to 2 . Suppose that we are given an input $M$ to the $\ell$-consecutive ones problem with $n$ rows and $m$ columns. (We will assume that $n \geq 3 \ell$.) We will show how to construct an input $S, c_{1}, \ldots, c_{m+\left(\ell_{-1}^{n}\right)}$ to the restricted $\ell$-phylogeny problem such that the phylogeny input has a restricted $\ell$-phylogeny if and only if the rows of $M$ can be permuted in such a way that for each column in the resulting matrix there are at most $\ell$ sequences of consecutive ones. 
The phylogeny input is constructed as follows. Let $M^{\prime}$ be a matrix derived from $M$ by replacing the zeroes in each column of $M$ with integers in the range $2, \ldots, n+1$ in such a way that each column of $M^{\prime}$ has at most one accurance of each integer in the range $2, \ldots, n+1$. The species set $S$ will have $n$ species one for each row of $M^{\prime}$. For $j$ in the range $1 \leq j \leq m$ character $c_{j}$ will map the species corresponding to row $r$ to the entry in column $j$ of row $r$ of $M^{\prime}$. We will define the remaining $\left(\begin{array}{c}n \\ \ell-1\end{array}\right)$ characters as follows. For $j$ in the range $1, \ldots,\left(\begin{array}{c}n \\ \ell-1\end{array}\right)$ we will have $R_{c_{j+m}}=\{0,1\}$. We will let $S_{j}$ denote the $j$ th size- $(\ell-1)$ subset of $S$ and we will set $c_{j+m}(s)=1$ for $s \in S_{j}$ and $c_{j+m}(s)=0$ for $s \notin S_{j}$.

$\rightarrow$ Suppose that $T$ is a restricted $\ell$-phylogeny for $S, c_{1}, \ldots, c_{m+\left(\ell_{-1}^{n}\right)}$. Using Fact 1 , we can assume that each species in $S$ is the label of exactly one node in $T$. Let $V=\left\{v_{1}, \ldots, v_{\ell-1}\right\}$ be any set of $\ell-1$ vertices of $T$ and let $j$ be the integer such that the species labeling the vertices in $V$ correspond to the set $S_{j}$. Observe that the graph obtained by removing the vertices in $V$ from $T$ has at most $\ell$ connected components (otherwise, the set of vertices $c_{j+m}^{-1}(0)$ form more than $\ell$ connected components in $T$, so $T$ is not an $\ell$-phylogeny). We will show that every node in $T$ has degree at most 2 . Suppose instead that $T$ has a vertex, $v_{1}$, of degree greater than or equal to 3 . We will show that there are $\ell-2$ other vertices, $v_{2}, \ldots, v_{\ell-1}$ such that the graph obtained by removing the vertices in $V=$ $\left\{v_{1}, \ldots, v_{\ell-1}\right\}$ from $T$ has at least $\ell+1$ connected components. This will be a contradiction, so we will conclude that every node in $T$ has degree at most 2. To show that $v_{2}, \ldots, v_{\ell-1}$ exist, note that the subgraph of $T$ formed by removing vertex $v_{1}$ has at least 3 connected components. Furthermore, if any subgraph $T^{\prime}$ of $T$. that is formed by removing up to $\ell-1$ vertices has fewer than $\ell+1$ connected components, it is possible to remove a vertex so as to increase the number of connected componentst. Let $v_{2}$ be a vertex such that removing $v_{2}$ from $T-v_{1}$ increases the number of connected components. Similarly, let $v_{3}$ be a vertex such that removing $v_{3}$ from $T-\left\{v_{1}, v_{2}\right\}$ increases the number of connected components. Continuing this process we identify $v_{2}, \ldots, v_{\ell-1}$. We have now shown that $T$ is a path. It follows that we can arrange the rows of $M$ in the order that the corresponding species occur on path $T$ and that, in such an arrangement, each column has at most $\ell$ sequences of consecutive ones.

$\leftarrow$ Suppose that $p=\left\{p_{1}, \ldots, p_{n}\right\}$ is a permutation of $\{1, \ldots, n\}$ such that when the rows of $M$ are permuted according to $p$ each column has at most $\ell$ sequences of consecutive ones. Let $T$ be a path consisting of the species in $S$, arranged according to permutation $p$. Then $T$ is a restricted $\ell$-phylogeny for $\left.S, c_{1}, \ldots, c_{n+(\ell-1}^{n}\right) \cdot \mathbf{\square}$

$\dagger$ To see this, note that (since $n \geq 3 \ell$ ) $T^{\prime}$ has some connected component with more than 2 vertices. 


\section{Two-Character Phylogeny}

In this section we show that for $k=2$ the phylogenetic number problem can be solved in polynomial time. We start by proving the following fact.

Fact 9: If a phylogeny input $S, c_{1}, c_{2}$ has an $\ell$-phylogeny then it has a restricted $\ell$-phylogeny $T$ in which each species in $S$ is the label of exactly one node and for each character $j \in\{1,2\}$ and each state $i \in R_{c_{j}}$, at most one of the connected components in the subgraph of $T$ induced by the set of vertices $c_{j}^{-1}(i)$ has more than one vertex.

Proof: Suppose that $T^{\prime}$ is a an $\ell$-phylogeny for $S_{1} c_{1}, c_{2}$. We start by showing that $S, c_{1}, c_{2}$ has a restricted $\ell$-phlogeny in which each species in $S$ is the label of exactly one node. We can assume that each species is the label of at most one node of $T^{\prime}$ (if not, combine branches). Now, suppose that a species $s \notin S$ is the label of some node of $T^{\prime}$. We can assume that this node, $v$, is an internal node of $T^{\prime}$ (otherwise delete it). Let $U_{1}$ be the set of neighbors $u$ of $v$ such that $c_{1}(u)=c_{1}(v)$. Let $U_{2}$ be the the set of neighbors $u$ of $v$ such that $c_{2}(u)=c_{2}(v)$. Note that $U_{1} \cap U_{2}=$ since $s$ is the only species that can label a node in $U_{1} \cap U_{2}$ and $v$ is the only node with label $s$. Let $U_{3}$ be the set of neighbors of $v$ that are not in $U_{1}$ or $U_{2}$. We can form a new $\ell$ phylogeny for $S, c_{1}, c_{2}$ by deleting node $v$, connecting the vertices in $U_{1}$ in a path, connecting the vertices in $U_{2}$ in a path, connecting the vertices in $U_{3}$ in a path, and connecting some node from $U_{1}$ to some node in $U_{2}$ and some node from $U_{2}$ to some node from $U_{3}$.

We have now shown that $S, c_{1}, c_{2}$ has a restricted $\ell$-phylogeny in which each species in $S$ is the label of exactly one node. Let $T$ be such an $\ell$-phylogeny. Suppose that for character $j \in\{1,2\}$ and state $i \in R_{c_{j}}, C$ and $C^{\prime}$ are two non-singleton connected components in the subgraph of $T$ induced by the set of vertices $c_{j}^{-1}(i)$. Let $c \in C$ and $c^{\prime} \in C^{\prime}$ be vertices such that the path connecting $c$ to $c^{\prime}$ in $T$ does not include any other vertices in $C$ or $C^{\prime}$. (Note that $c$ and $c^{\prime}$ are uniquely defined.) For every neighbor $v$ of $c$ in $C$ note that the path between $v$ and $c^{\prime}$ passes through vertex $c$. Remove the edge $(v, c)$ from $T$ and add the edge $\left(v, c^{\prime}\right)$. Note that the resulting tree is an $\ell$-phylogeny for $S, c_{1}, c_{2}$. (To see this, note that since the species labeling $v$ is different from the species labeling $c$, the character other than character $j$ disagrees on $v$ and $c$.) 0

In this section, we will represent the phylogeny input $S, c_{1}, c_{2}$ as a bipartite graph. One set of vertices in the graph will be the set $R_{c_{1}}$ and the other set of vertices in the graph will be the set $R_{c_{2}}$. For $i \in R_{c_{1}}$ and $j \in R_{c_{2}}$ the edge $(i, j)$ will be present in the graph if and only if $S$ contains a species $s$ such that $c_{1}(s)=i$ and $c_{2}(s)=j$. We will use the notation $d(u)$ to denote the degree of a vertex $u$ in this graph. We will define a special $\ell$-coloring of the graph to be a coloring of the edges with the colors white, blue, red, and purple such that each vertex $i$ in $R_{c_{1}}$ has $\max (0, d(i)-\ell+1)$ of its neighboring edges colored either red or purple and the rest of its neighboring edges colored either white or blue and each vertex $j$ in $R_{c_{2}}$ has $\max (0, d(j)-\ell+1)$ of its neighboring 
edges colored either blue or purple and the rest of its neighboring edges colored either white or red. (Intuitively, think of each edge as starting out white. Then each vertex $i$ in $R_{c_{1}}$ adds red color to $\max (0, d(i)-\ell+1)$ of its neighboring edges and each vertex $j$ in $R_{c_{2}}$ adds blue color to $\max (0, d(j)-\ell+1)$ of its neighboring edges. Edges that get colored both red and blue in this process become purple.) We will prove the following lemma.

Lemma 10: A phylogeny input $S, c_{1}, c_{2}$ has an $\ell$-phylogeny if and only if the corresponding bipartite graph has a special $\ell$-coloring with no purple cycle.

Proof: First, suppose that the input $S, c_{1}, c_{2}$ has an $\ell$-phylogeny. By Fact 9 it has a restricted $\ell$-phylogeny $T$ in which each species in $S$ is the label of exactly one node and for each character $h \in\{1,2\}$ and each state $i \in R_{c_{h}}$, at most one of the connected components in the subgraph of $T$ induced by the set of vertices $c_{h}^{-1}(i)$ has more than one vertex. Construct a special $\ell$-coloring as follows. For each vertex $i \in R_{c_{1}}$ let $C_{i}$ be the largest connected component in the subgraph of $T$ induced by the set of vertices $c_{1}^{-1}(i)$. Arbitrarily choose $\max (0, d(i)-\ell+1)$ of the vertices in $C_{i}$ and add red color to the corresponding edges in the graph. For each vertex $j \in R_{c_{2}}$ let $C_{j}$ be the largest connected component in the subgraph of $T$ induced by the set of vertices $c_{2}^{-1}(j)$. Arbitrarily choose $\max (0, d(j)-\ell+1)$ of the vertices in $C_{j}$ and add blue color to the corresponding edges in the graph. We will now argue that the special $\ell$-colored graph has no purple cycle. Suppose instead that the special $\ell$-colored graph has a purple cycle consisting of the edges $\left(i_{1}, j_{1}\right),\left(i_{2}, j_{1}\right),\left(i_{2}, j_{2}\right), \ldots,\left(i_{m}, j_{m}\right),\left(i_{1}, j_{m}\right)$. Then, by construction, there is a path in $T$ between the species $\left(i_{1}, j_{1}\right)$ and the species $\left(i_{2}, j_{1}\right)$ which is contained in $C_{j_{1}}$. Similarly, there is a path in $T$ between the species $\left(i_{2}, j_{1}\right)$ and the species $\left(i_{2}, j_{2}\right)$ which is contained in $C_{i_{2}}$. These paths intersect exactly at the species $\left(i_{2}, j_{1}\right)$. Continuing in this manner, we construct a cycle in $T$, which contradicts the fact that $T$ is a phylogeny.

Next, suppose that the graph has a special $\ell$-coloring with no purple cycle. Construct an $\ell$-phylogeny $T$ as follows. The nodes of $T$ are the species in $S$. For each vertex $i \in R_{c_{1}}$ let $C_{i}$ be the set of species in $c_{1}^{-1}(i)$ such that the corresponding edges in the graph have red color. Add a path to $T$ which traverses the nodes in $C_{i}$. All of the species on this path have the same state in character 1. Also, these species correspond to red edges in the special $\ell$-coloring. For the purpose of the proof, we will think of the corresponding nodes in the path as having red color. For each vertex $j \in R_{c_{2}}$ let $C_{j}$ be the set of species in $c_{2}^{-1}(j)$ such that the corresponding edges in the graph have blue color. Add a path to $T$ which traverses the nodes in $C_{j}$. All of the species on this path have the same state in character 2. Also, these species correspond to blue edges in the special $\ell$-coloring. For the purpose of the proof, we will think of the corresponding nodes in the path as having blue color. We will now argue that $T$ has no cycle. Suppose instead that $T$ has a cycle. Note by construction that every edge in the cycle either fixes character 1 or fixes character 2 (but not both). For example, the cycle might look like $\left(i_{1}, j_{1}\right),\left(i_{1}, j_{2}\right),\left(i_{1}, j_{3}\right),\left(i_{2}, j_{3}\right),\left(i_{2}, j_{4}\right),\left(i_{2}, j_{5}\right),\left(i_{1}, j_{5}\right)$. Let $\left(x_{1}, y_{1}\right), \ldots,\left(x_{m}, y_{m}\right)$ be the sequence of nodes that we get when we traverse the 
nodes in the cycle in order, skipping any node such that the edge into the node fixes the same character as the edge out of the node. (For the above example, we get the sequence $\left(i_{1}, j_{1}\right),\left(i_{1}, j_{3}\right),\left(i_{2}, j_{3}\right),\left(i_{2}, j_{5}\right),\left(i_{1}, j_{5}\right)$.) Each species $\left(x_{a}, y_{a}\right)$ is colored purple in $T$, so each edge $\left(x_{a}, y_{a}\right)$ is colored purple in the graph. (To see that species $\left(x_{a}, y_{a}\right)$ is colored purple in $T$, note that it is part of a path fixing the state of character 1 (hence, red color is added). It is also part of a path fixing the state of character 2 (hence, blue color is added).) Finally, we observe that the edges $\left(x_{a}, y_{a}\right)$ form a cycle in the graph, which contradicts the fact that the graph has no purple cycle. We conclude that $T$ has no cycle. If $T$ is disconnected, we arbitrarily add edges making it into a tree. $\square$

We now present a polynomial-time algorithm that takes as input an integer $\ell$ and a bipartite graph $G$ and determines whether $G$ has a special $\ell$-coloring with no purple cycle. The algorithm proceeds by considering a sequence of special $\ell$ colored graphs $G_{0}, G_{1}, \ldots$ Graph $G_{0}$ is an arbitrary special $\ell$-coloring of the graph $G$. For $t \geq 1, G_{t}$ is constructed by modifying the coloring in $G_{t-1}$. We will use the notation $\mathcal{E}\left(G_{t}\right)$ to denote the set of edges that are contained in some purple cycle in $G_{t}$. When the algorithm considers the graph $G_{t-1}$ it will either produce a graph $G_{t}$ such that $\mathcal{E}\left(G_{t}\right) \subset \mathcal{E}\left(G_{t-1}\right)$ or it will terminate with the answer "no". If the aigorithm ever produces a graph $G_{t}$ such tha $\mathcal{E}\left(G_{t}\right)=\emptyset$ it will terminate with the answer "yes".

We now show how to construct the graph $G_{t}$ from $G_{t-1}$ (or to terminate with the answer "no"). Fix an edge $e \in \mathcal{E}\left(G_{t-1}\right)$. The procedure will consider a sequence of special $\ell$-colored graphs $G_{0}^{\prime}=G_{t-1}, G_{1}^{\prime}, G_{2}^{\prime}, \ldots$ For each graph $G_{j}^{\prime}$ in the sequence, $e$ will be a member of $\mathcal{E}\left(G_{j}^{\prime}\right)$. For each graph $G_{j}^{\prime}$, let $P\left(G_{j}^{\prime}\right)$ be the graph that is obtained by considering all of the purple edges in $G_{j}^{\prime}$ (and no other edges) and let $\left(U_{e}\left(G_{j}^{\prime}\right), V_{e}\left(G_{j}^{\prime}\right)\right)$ be the vertices of the connected component in $P\left(G_{j}^{\prime}\right)$ that contains $e$. Let $M_{e}\left(G_{j}^{\prime}\right)$ be the set of edges in the connected component in $P\left(G_{j}^{\prime}\right)$ that contains $e$. To transform $G_{j}^{\prime}$ into $G_{j+1}^{\prime}$ the algorithm may make one e-move in which it either selects a vertex $u \in U_{e}\left(G_{j}^{\prime}\right)$ and transfers the red color from one edge adjacent to $u$ to another edge adjacent to $u$ that does not already have red color or the procedure selects a vertex $v \in V_{e}\left(G_{j}^{\prime}\right)$ and transfers the blue color from one edge adjacent to $v$ to another edge adjacent to $v$ that does not already have blue color. The move is legal if and only if $\mathcal{E}\left(G_{j+1}^{\prime}\right) \subseteq \mathcal{E}\left(G_{j}^{\prime}\right)$. Such a move is called a finishing move if $\mathcal{E}\left(G_{j+1}^{\prime}\right) \subset \mathcal{E}\left(G_{j}^{\prime}\right)$. It is called an $e$-continuing move if it is not finishing, but $M_{e}\left(G_{j+1}^{\prime}\right) \subset M_{e}\left(G_{j}^{\prime}\right)$. When it considers the special $\ell$-colored graph $G_{j}^{\prime}$, the algorithm checks every possible $e$-move. If it finds a legal $e$-move, it constructs $G_{j+1}^{\prime}$ by making this move. If the move is finishing, then the procedure returns the graph $G_{t}=G_{j+1}^{\prime}$. If the move is not finishing, but it is e-continuing, the procedure now considers the graph $G_{j+1}^{\prime}$. (Note that in this case $\mathcal{E}\left(G_{j+1}^{\prime}\right)=\mathcal{E}\left(G_{j}^{\prime}\right)$ so $e \in \mathcal{E}\left(G_{j}^{\prime}\right)$.) If there are no legal $e$-moves that are finishing or $e$-continuing, the algorithm terminates with the answer "no". Note that at most $\left|M_{e}\left(G_{0}^{\prime}\right)\right|$ continuing moves can be made, so the procedure terminates in polynomial time.

The correctness of the algorithm follows from the following lemma. 
Lemma 1: If a bipartite graph $G$ has a special $\ell$-coloring with no purple cycle and $H$ is a special $\ell$-coloring of $G$ with $e \in \mathcal{E}(H)$ then there is a legal $e$-move from $H$ that is either finishing or $e$-continuing.

Proof: Let $G_{e}$ be the subgraph of $G$ induced by $U_{e}(H) \cup V_{e}(H)$ and let $S_{e}$ denote the set of edges in $G_{e}$. We wish to compute an upper bound for $\left|S_{e}\right|$. To do so, let $d^{\prime}(w)$ denote the degree of vertex $w$ in graph $G_{e}$. Since $G$ has a special $\ell$-coloring with no purple cycle, $G_{e}$ has a special $\ell$-coloring with no purple cycle. Let $H_{e}^{\prime}$ be such a special $\ell$-coloring of $G_{e}$. The number of edges with red color added in $H_{e}^{\prime}$ is at least $\sum_{u \in U_{e}(H)}\left(d^{\prime}(u)-\ell+1\right)$. The number of edges with blue color added is at least $\sum_{v \in V_{e}(H)}\left(d^{\prime}(v)-\ell+1\right)$. The number of purple edges (which have both red color and blue color) is at most $\left|U_{e}(H)\right|+\left|V_{e}(H)\right|-1$. Hence,

$\left|S_{e}\right| \geq \sum_{u \in U_{e}(H)}\left(d^{\prime}(u)-\ell+1\right)+\sum_{v \in V_{e}(H)}\left(d^{\prime}(v)-\ell+1\right)-\left(\left|U_{e}(H)\right|+\left|V_{e}(H)\right|-1\right)$

and therefore $\left|S_{e}\right|<\ell\left(\left|U_{e}(H)\right|+\left|V_{e}(H)\right|\right)$.

Now cunsider $H$. Let $S_{1}$ be the set of edges that are adjacent to vertices in $U_{e}$ and do not have red color. Let $S_{2}$ be the set of edges that are adjacent to vertices in $V_{e}$ and do not have blue color. Suppose that some edge $e^{\prime}$ is in $S_{1} \cap S_{2}$. Let $e^{\prime \prime}$ be a purple edge that is adjacent to $e^{t}$. Clearly, the $e$-move that transfers color from $e^{\prime \prime}$ to $e^{\prime}$ is legal. Suppose that it is not finishing and let $H^{\prime}$ be the graph obtained from $H$ by making this move. Then $M_{e}\left(H^{\prime}\right) \subseteq M_{e}(H)-\left\{e^{\prime \prime}\right\}$. Hence, the move is $e$-continuing.

Suppose instead that $S_{1} \cap S_{2}=\emptyset$. Every vertex $w \in U_{e}(H) \cup V_{e}(H)$ has $d(w) \geq \ell$. (If $d(w)<\ell$ then $w$ will not add color to its neighboring edges in any special $\ell$-coloring of $G$ so $w$ will not be in the connected component containing $e$ in $P(H)$.) Therefore $\left|S_{1}\right| \geq \sum_{u \in U_{e}(H)}(\ell-1)$ and $\left|S_{2}\right| \geq \sum_{v \in V_{e}(H)}(\ell-1)$. Let $S_{3}$ be the set of purple edges with endpoints in $U_{e}(H) \cup V_{e}(H)$. Note that $\left|S_{3}\right| \geq\left|U_{e}\right|+\left|V_{e}\right| . S_{3}$ is disjoint from $S_{1}$ and $S_{2}$ so $\left|S_{1} \cup S_{2} \cup S_{3}\right| \geq \ell\left(\left|U_{e}(H)\right|+\left|V_{e}(H)\right|\right)$. We conclude that some edge in $S_{1}$ or $S_{2}$ must have an endpoint outside of $G_{e}$.

Without loss of generality, assume that there is an edge $e^{\prime} \in S_{1}$ that has endpoint $u \in U_{e}(H)$ and its other endpoint, $v$, outside of $V_{e}(H)$. There are two cases. Suppose that $u$ is contained in a purple cycle in $H$. Let $(u, w)$ be an edge in such a cycle. Consider the $e$-move that transfers color from $(u, w)$ to $e^{t}$. This move is legal. (Since $v$ is not in $V_{e}(H)$ no purple cycles are created by the move.) Let $H^{\prime}$ be the graph obtained from $H$ by making this move. $\mathcal{E}\left(H^{\prime}\right) \subseteq \mathcal{E}(H)-\{(u, w)\}$, so the move is finishing. Suppose instead that $u$ is not contained in a purple cycle in $H$. Let $(u, w)$ be the first edge on the unique path from $u$ to $e$ in $P(H)$. Consider the legal $e$-move that transfers color from $(u, w)$ to $e^{\prime}$. Suppose that it is not finishing and let $H^{\prime}$ be the graph obtained from $H$ by making this move. Then $M_{e}\left(H^{\prime}\right) \subseteq M_{e}(H)-\{(u, w)\}$. Hence, the move is $e$-continuing. 
In Lemma 10 we showed that a phylogeny input $S, c_{1}, c_{2}$ has an $\ell$-phylogeny if and only if the corresponding bipartite graph has a special $\ell$-coloring with no purple cycle. We then described a polynomial time algorithm that takes as input an integer $\ell$ and a bipartite graph $G$ and determines whether $G$ has a special $\ell$-coloring with no purple cycle. Hence, we have shown that there is a polynomial time algorithm that takes input $\ell$ and a phylogeny input $S, c_{1}, c_{2}$ and determines whether the phylogeny input has an $\ell$-phylogeny. (In fact, our algorithm constructs an $\ell$-phylogeny if one exists.) Using binary search (or even linear search) on $\ell$, we obtain a polynomial-time algorithm that takes as input a phylogeny input $S, c_{1}, c_{2}$ and determines the phylogenetic number of the input. Hence, we have proved the following theorem.

Theorem 12: The phylogenetic number problem can be solved in polynomial time for $k=2$.

Unfortunately, Fact 9 no longer holds if we add a third character $c_{3}$. Hence, our approach does not solve the phylogenetic number problem (or even the $\ell$ phylogeny problem) for fixed $k \geq 3$. (To see that Fact 9 does not hold for $k>2$, consider the 3 -species 3 -character input $\{100,010,001\}$. One can construct a 1-phylogeny for this input by attaching each species to the new species 000 . However, the input does not have a restricted 1-phylogeny.)

\section{Phylogeny With a Fixed Number of States}

In subsection 6.1 we show that the fixed-topology phylogenetic number problem can be solved in polynomial time for fixed $r$. On a related note, we show that if $r$ is fixed, there is a polynomial-delay algorithm for listing fixed-topology $\ell$ phylogenies. In subsection 6.2 we show that for fixed $r \geq 2$ and fixed $\ell \geq 3$ the restricted $\ell$-phylogeny problem is NP-hard. (This result follows from a more general result. Namely, we show that the restricted $\left(\ell_{1}, \ell_{2}\right)$-phylogeny problem is NP-hard for fixed $\ell_{1} \geq 2$ and $\ell_{2} \geq 2$ as long as one of $\ell_{1}, \ell_{2}$ is greater than 2.)

\subsection{Fixed-Topology Phylogeny with a Fixed Number of States}

In this subsection we prove the following theorem.

Theorem 13: The fixed-topology phylogenetic number problem can be solved in polynomial time for fixed $r$.

It suffices to consider each character independently. We are given an input tree $T$ with each of its $n$ leaves labeled by a state in the range $\{1, \ldots, r\}$. We wish to label the internal nodes of $T$ to construct a phylogeny with the smallest possible phylogenetic number. We root the tree at an arbitrary node, constructing the child and parent pointers. The choice of root will not affect the phylogenetic number of the tree. 
For a given character, this problem can be solved by a two-pass algorithm: once up the tree and once down. In the upward phase, for each node $v$, and for each vector in the set

$$
\left\{\left(i, \ell_{1}, \ldots, \ell_{r}\right) \mid(1 \leq i \leq r) \text { and } 1 \leq \ell_{j} \leq n \text { for } 1 \leq j \leq r\right\}
$$

we construct, if possible, a labeling of the nodes in the subtree rooted at $v$ such that $v$ is labeled with state $i$ and, in the subtree rooted at $v$, the subgraph induced by nodes labeled $j$ has exactly $\ell_{j}$ connected components. We call such a labeling a configuration of the subtree rooted at $v$, or a configuration of $v$ for short. If there are no leaves in this subtree labeled $j$ for some $j \in\{1, \ldots, r\}$, then we have $\ell_{j}=0$ for all configurations (there are no connected components labeled $j$ in the subtree rooted at $v$ ).

There are $O\left(r n^{r}\right)$ possible configurations for the subtree rooted at any node, with one possible configuration for each leaf. Once the possible configurations have been constructed for the children of a node, we can construct the possible configurations for the parent by combining configurations of the children incrementally. Consider the first two children $v_{1}$ and $v_{2}$ of parent node $v$. For each pairing of a configuration for $v_{1}$ with a configuration for $v_{2}$, we construct $r$ configurations for the subtree consisting of parent node $v$ and the sibtrees rooted at children $v_{1}$ and $v_{2}$, one configuration for each possible labeling of the parent $v$. If node $v$ is labeled $i$, and the configurations of $v_{1}$ and $v_{2}$ are represented by the vectors $\left(i_{1}, \ell_{11}, \ell_{12}, \ldots, \ell_{1 r}\right)$ and $\left(i_{2}, \ell_{21}, \ell_{22}, \ldots, \ell_{2 r}\right)$ respectively, then the resulting configuration is $\left(i, \ell_{1}, \ell_{2}, \ldots, \ell_{r}\right)$ where $\ell_{j}=\ell_{1 j}+\ell_{2 j}$ for all $j \neq i$, and $\ell_{i}=\ell_{1 i}+\ell_{2 i}+1-m$, where $m \in\{0,1,2\}$ is the number of children (considering only $v_{1}$ and $v_{2}$ ) which are labeled $i$. That is, the number of components of state $j$ is the sum of the number of components in each child for most states. The only state that can differ is the state with which node $v$ is labeled $(i)$. In this case, if neither $v_{1}$ nor $v_{2}$ is labeled $i$, then we create a new component of state $i$ (the node $v$ ) in addition to the components present in the children. If exactly one child is labeled $i$, then the label of node $v$ becomes part of that component. If both $v_{1}$ and $v_{2}$ are labeled $i$, then one component of state $i$ from each child can merge through node $v$, and the number of components in the combination is one fewer than the sum.

Whenever a new possible configuration is achieved through a combination of configurations in the two children, it is recorded along with pointers to the configurations of $v_{1}$ and $v_{2}$ that achieve this phylogenetic configuration. Although there are $r^{2} n^{2 r}$ ways to pair up the configurations of two children, there can be at most $r n^{r}$ configurations for the parent. If a configuration is achieved multiple ways, we only remember one way.

After computing the $O\left(r n^{r}\right)$ configurations for the subtree consisting of node $v$ with the subtrees rooted at $v_{1}$ and $v_{2}$ (call this tree $T^{\prime}$ ), we now add child $v_{3}$. The compuation is almost the same as before. Let possible configurations for $T^{\prime}$ and the subtree rooted at $v_{3}$ be represented by vectors $\left(i, \ell_{1}^{\prime}, \ell_{2}^{\prime}, \ldots, \ell_{r}^{\prime}\right)$ and $\left(j, \ell_{31}, \ell_{32}, \ldots, \ell_{3 r}\right)$ respectively. Then the combined configuration is $\left(i, \ell_{1}, \ell_{2}, \ldots, \ell_{r}\right)$ where $\ell_{k}=\ell_{k}^{\prime}+\ell_{3 k}$ for all $k$, unless $i=j$. In 
this case, we have $\ell_{i}=\ell_{i}^{\prime}+\ell_{3 i}-1$ because one component of state $i$ from the subtree rooted at $v_{3}$ can connect to components of state $i$ from the other children through the parent $v$.

Each child of node $v$ is added in this way until we have computed the $O\left(r n^{r}\right)$ possible configurations for the entire subtree rooted at node $v$. We continue up the tree until we have computed all possible configurations for the root. This computation takes $O\left(r^{2} n^{2 r+1}\right)$ time. We then pick a possible configuration with the minimum phylogenetic number and go down the tree generating labels by following the pointers to the subconfigurations that achieve the optimal configuration.

The above algorithm makes it clear that if $r$ is fixed, there is a polynomialdelay algorithm for listing fixed-topology $\ell$-phylogenies.

\subsection{Restricted Phylogeny with a Fixed Number of States}

In this subsection we show that for fixed $r \geq 2$ and fixed $\ell \geq 3$ the restricted $\ell$-phylogeny problem is NP-hard.

We start by proving the following more general theorem.

Theorem 14: The restricted $\left(\ell_{1}, \ell_{2}\right)$-phylogeny problem is NP-hard far fixed $\ell_{1} \geq 2$ and $\ell_{2} \geq 2$ as long as one of $\ell_{1}, \ell_{2}$ is greater than 2 .

Proof: Without loss of generality, assume that $\ell_{1} \geq \ell_{2}$. The reduction is from the 2 -consecutive ones problem.

Let $M$ be the matrix in the input to the 2-consecutive ones problem. Let $n^{\prime}$ denote the number of rows of $M$ and $m$ denote the number of columns of $M$. (We will assume that $n^{i} \geq 3 \ell_{2}$.) We will show how to construct an inpui to the restricted $\left(\ell_{1}, \ell_{2}\right)$-phylogeny problem such that the phylogeny input has a restricted $\left(\ell_{1}, \ell_{2}\right)$-phylogeny if and only if the rows of $M$ can be permuted in such a way that for each column in the resulting matrix there are at most 2 sequences of consecutive ones.

The phylogeny input is constructed as follows. Let $M^{\prime}$ be a matrix derived from $M$ by adding $2\left(\ell_{2}-2\right)$ rows to the bottom of $M$. The entries in the $\left(n^{\prime}+i\right)$ th row are equal to 0 for odd $i>0$ and are equal to one for even $i>0$. Let $n$ denote $n^{\prime}+2\left(\ell_{2}-2\right)$. Note that $M^{\prime}$ has $n$ rows. The species set $S=\left\{s_{1}, \ldots, s_{n}\right\}$ will have $n$ species. Species $s_{i}$ will correspond to row $i$ of $M^{\prime}$. Let $k_{1}$ denote $\left(\begin{array}{c}n \\ \ell_{2}-1\end{array}\right)$. Let $k_{2}$ denote $\left(\begin{array}{c}n^{\prime} \\ \ell_{2}-1\end{array}\right)$. Let $k_{3}$ denote $\max \left(0, n-n^{\prime}-1\right)$. Let $k$ denote $m+k_{1}+k_{2}+k_{2} k_{3}$. The input to the phylogeny problem will be $S, c_{1}, \ldots, c_{k}$. The characters $c_{1}, \ldots, c_{k}$ will be defined as follows:

1. (Characters that describe $M^{\prime}$ ) For $j$ in the range $1 \leq j \leq m$ character $c_{j}$ will map species $s_{i}$ to the entry in column $j$ of row $i$ of $M^{\prime}$.

2. (Characters that make every phylogeny a path) For $j$ in the range $1 \leq j \leq$ $k_{1}$ let $S_{j}$ denote the $j$ th size- $\left(\ell_{2}-1\right)$ subset of $S$. We set $c_{m+j}(s)=0$ for $s \in S_{j}$ and $c_{m+j}(s)=1$ for $s \notin S_{j}$. 
3. (Characters that place $s_{n}$ at one end of the path) For $j$ in the range $1 \leq$ $j \leq k_{2}$ let $S_{j}^{\prime}$ denote the $j$ th size- $\left(\ell_{2}-1\right)$ subset of $\left\{s_{1}, \ldots, s_{n^{\prime}}\right\}$. We set $c_{m+k_{1}+j}(s)=0$ for $s \in S_{j}^{\prime}$ and $c_{m+\dot{k}_{1}+j}\left(s_{n}\right)=0$ and $c_{m+k_{1}+j}(s)=1$ for every other species $s$.

4. (Characters that place $s_{n^{\prime}+1}, \ldots, s_{n^{2}}$ consecutively at the end of the path) For $j$ in the range $1 \leq j \leq k_{2}$ and $i$ in the range $1 \leq i \leq k_{3}$ let $m^{\prime}$ denote $m+k_{1}+k_{2}+(i-1) k_{2}+j$. We set $c_{m^{\prime}}\left(s_{r}\right)=0$ for $s_{r} \in S_{j}^{\prime}$ and $c_{m^{\prime}}\left(s_{r}\right)=1$ for $s_{r} \in\left\{s_{1}, \ldots, s_{n^{\prime}}\right\}-S_{j}^{\prime}$. Furthermore, we set $c_{m^{\prime}}\left(s_{n^{\prime}+1}\right)=$ $\cdots=c_{m^{\prime}}\left(s_{n-i-1}\right)=1$ and we set $c_{m^{\prime}}\left(s_{n-i}\right)=\cdots=c_{m^{\prime}}\left(s_{n}\right)=0$.

$\rightarrow$ Suppose that $T$ is a restricted $\left(\ell_{1}, \ell_{2}\right)$-phylogeny for $S, c_{1}, \ldots, c_{k}$. Using Fact 1, we can assume that each species in $S$ is the label of exactly one node in $T$. Following the proof of Theorem 8 , we can show that every node in $T$ has degree at most 2. That is, $T$ is a path. If $n=n^{\prime}$ (i.e., $\ell_{2}=2$ ) then it follows that we can arrange the rows of $M$ in the order that the species occur in path $T$ and that, in such an arrangement, each column has at most 2 sequences of consecutive ones. Suppose instead that $n>n^{\prime}$. We will now show that the node labeled $s_{n}$ has degree 1. Suppose instead that it has degree 2. We argue as in the proof of Theorem 8 that there is a size- $\left(\ell_{2}-1\right)$ set $S^{\prime} \subseteq\left\{s_{1}, \ldots, s_{n^{\prime}}\right\}$ such that if $s_{n}$ and the species in $S^{\prime}$ are removed from $T$, the resulting subgraph has at least $\ell_{2}+1$ connected components. Let $j$ be the integer such that $S^{\prime}=S_{j}^{\prime}$. Then the set of vertices $c_{m+k_{1}+j}^{-1}(1)$ form more than $\ell_{2}$ connected components in $T$, which is a contradiction. We conclude that the node labeled $s_{n}$ is an endpoint of the path. For $i$ in the range $1 \leq i \leq k_{3}$ we will now argue that the node labeled $s_{n-i}$ is adjacent to a node with a label in $\left\{s_{n-i+1}, \ldots, s_{n}\right\}$. Suppose that this is not the case. We argue as in the proof of Theorem 8 that there is a size$\left(\ell_{2}-1\right)$ set $S^{\prime} \subseteq\left\{s_{1}, \ldots, s_{n^{\prime}}\right\}$ such that if the species in $S^{\prime} \cup\left\{s_{n-i}, \ldots, s_{n}\right\}$ are removed from $T$ then the resulting subgraph has at least $\ell_{2}+1$ connected components. Let $j$ be the integer such that $S^{\prime}=S_{j}^{\prime}$. Then the set of vertices $c_{m+k_{1}+k_{2}+(i-1) k_{2}+j}^{-1}(1)$ form more than $\ell_{2}$ connected components in $T$, which is a contradiction. We conclude that $T$ is a path consisting of the species in $\left\{s_{1}, \ldots, s_{n^{\prime}}\right\}$ (in some order) followed by $s_{n^{\prime}+1}, \ldots, s_{n}$. It follows that we can arrange the rows of $M$ in the order that the species occur in path $T$ and that, in such an arrangement, each column has at most 2 sequences of consecutive ones.

$\leftarrow$ Suppose that $p=\left\{p_{1}, \ldots, p_{n}^{\prime}\right\}$ is a permutation of $\left\{1, \ldots, n^{\prime}\right\}$ such that when the rows of $M$ are permuted according to $p$ each column has at most 2 sequences of consecutive ones. If $\ell_{2}=2$ then let $T$ be the path consisting of the species in $\left\{s_{1}, \ldots, s_{n^{\prime}}\right\}$, arranged according to $p . T$ is a restricted $(3,2)$-phylogeny for $S, c_{1}, \ldots, c_{k}$. Hence, $T$ is a restricted $\left(\ell_{1}, \ell_{2}\right)$-phylogeny for $S, c_{1}, \ldots, c_{k}$. Suppose instead that $\ell_{2}>2$. Let $T$ be a path consisting of the species in $\left\{s_{1}, \ldots, s_{n^{\prime}}\right\}$, arranged according to permutation $p$, followed by $s_{n^{\prime}+1}, \ldots, s_{n}$. Then $T$ is a restricted $\left(\ell_{2}, \ell_{2}\right)$-phylogeny for $S, c_{1}, \ldots, c_{k}$. Hence, $T$ is a restricted $\left(\ell_{1}, \ell_{2}\right)$-phylogeny for $S, c_{1}, \ldots, c_{k}$.

Note that Theorem 14 has the following corollary. 
Corollary 15: For fixed $r \geq 2$ and fixed $\ell \geq 3$ the restricted $\ell$-phylogeny problem is NP-hard.

\section{Conclusions}

In this section we present some open problems. There are several restrictions of the parameters which yield problems for which the complexity is still open. Recall that $k$ is the number of characters, $r$ is the maximum number of states for any character, and $\ell$ is the phylogenetic number. It is unknown whether the following restricted versions of the $\ell$-phylogeny problem can be solved by polynomial-time algorithms:

1. Finding an $\ell$-phylogeny where the number $k$ of characters is a constant greater than 2 (for $\ell>1$ ),

2. Finding an $\ell$-phylogeny where the number $r$ of states per character is a constant.

3. For the case where $r=2$, determining whether an input has a $(1,2)$ phylogeny or a $(2,2)$ phylogeny. Recall that for $r=2$, the problem of finding a $(1,1)$-phylogeny is in $\mathcal{P}$, but finding a $(2,3)$-phylogeny is $\mathcal{N P}$. complete.

This paper also leaves open the problems of randomly generating phylogenies with constraints upon their phylogenetic number and approximation algorithms for the $\mathcal{N P}$-complete versions of the $\ell$-phylogeny problem. In particular, suppose that there exists a perfect phylogeny. For what $\ell$ can we find an $\ell$-phylogeny in polynomial time (with $\ell$ possibly a function of $k$ and $r$ )?

\section{Acknowledgements}

The work of Leslie Ann Goldberg, Paul Goldberg and Cynthia Phillips was supported by the U.S. Department of Energy under contract DE-AC04-76AL85000. Elizabeth Sweedyk was supported by the California Legislative Grant. The work of Tandy Warnow was supported by a National Science Foundation Young Investigator Award under contract CCR-9457800, and by the U.S. Department of Energy under contract DE-AC04-76AL85000. 


\section{References}

1. R. Agarwala and D. Fernandez-Baca, "Fast and Simple Algorithms for Perfect Phylogeny and Triangulating Colored Graphs", DIMACS TR\#94-51, 1994.

2. R. Agarwala, D. Fernández-Baca, "A Polynomial-Time Algorithm for the Perfect Phylogeny Problem when the Number of Character States is Fixed", procs. of the 34th annual Symposium on Foundations of Computer Science, 1993.

3. H. Bodlaender, M. Fellows, T. Warnow, "Two Strikes Against Perfect Phylogeny", procs. of the 19th International Congress on Automata, Languages and Programming (ICALP), pp. 273-287, Springer-Verlag Lecture Notes in Computer Science, 1992.

4. W.H.E. Day, "Computationally difficult parsimony problems in phylogenetic systematics," Journal of theoretical biology, 103: 429-438.

5. W.H.E. Day and D. Sankoff, "Computational complexity of inferring phylogenies by compatibility", Systematic Zoology, 35(2): 224-229, 1986.

7. Wm. Fitch, "Toward defining the course of evolution: minimum change for a specified tree topology", Syst. Zool., 20:406-416, 1971.

8. D.R. Fulkerson, D.A. Gross, "Incidence matrices and interval graphs", Pacific J. Math., 15 (3), 1965.

9. P.W. Goldberg, M.C. Golumbic, H. Kaplan, R. Shamir, "Four Strikes Against Physical Mapping of DNA", Tech. Rept. 287/93, Tel Aviv University, 1993.

10. S. Kannan and T. Warnow, "Inferring Evolutionary History from DNA Sequences", SIAM J. on Computing, Vol. 23, No. 4, August 1994.

11. S. Kannan and T. Warnow, "A fast algorithm for the computation and enumeration of perfect phylogenies", to appear, $A C M / S I A M$ Symposium on Discrete Algorithms, 1995.

12. F.R. McMorris, T. Warnow, T. Wimer, "Triangulating Colored Graphs", SIAM J. on Discrete Mathematics, Vol. 7, No. 2, pp. 296-306, 1994.

13. R.C. Prim, "Shortest Connection Networks and Some Generalisations", Bell System Tech. J., 36 1389-1401, 1957.

14. M.A. Steel, "The complexity of reconstructing trees from qualitative characters and subtrees", Journal of Classification, 9 91-116, 1992.

15. T. Warnow, "Efficient Algorithms for The Character Compatibility Problem", New Zealand Journal of Botany, Vol. 31, (1993), pp. 239-248.

This article was processed by the author using the $\mathcal{T E X}_{\mathrm{X}}$ macro package from SpringerVerlag. 